\title{
El programa "Cuerda pulsada" y su eficacia, para el aprendizaje de la guitarra, en estudiantes del primer año de secundaria, 2019
}

\section{The "Plucked String" program and its effectiveness, for learning the guitar, in high school freshmen, 2019}

\section{O programa "Plucked String" e sua eficácia, para aprender a tocar violão, nos calouros do ensino médio, 2019}

\author{
Gustavo Samuel Ruiz García \\ samuelruiz@,upeu.edu.pe \\ iD https://orcid.org/0000-0002-9450-2524 \\ Universidad Peruana Unión
}

Recibido: 24 de junio de 2021

Aceptado: 28 de diciembre 2021

\section{Resumen}

La presente investigación tiene el objetivo de determinar la eficacia del método cuerda pulsada, para el aprendizaje básico de la guitarra de manera grupal. Esta investigación es cuantitativa, de diseño preexperimental, realizada con 16 estudiantes. El instrumento fue validado por juicio de expertos y mediante el análisis estadístico: SPSS, versión 27, dando la confiabilidad respectiva. Los resultados muestran que el "método: cuerda pulsada" fue eficaz, para el aprendizaje básico de la guitarra de manera grupal; hubo una mejora significativa; en el pre test, el $75 \%$ de los estudiantes se encontraba en un nivel bajo $6.8 \%$, poseían un nivel muy bajo y el $18.3 \%$ se encontraban en un nivel regular. Después de aplicado el programa, el post test nos indica que un $25 \%$ poseía un nivel regular, un $43.8 \%$ estaba en el nivel bueno y un 25\% logró un nivel de excelencia. En conclusión, el método referido fue eficaz, para el aprendizaje musical y la ejecución de la guitarra.

Palabras claves: método, ejecución, guitarra, aprendizaje grupal.

\begin{abstract}
The present investigation has the objective of determining the effectiveness of the plucked string method, for the basic learning of the guitar in a group way. This research is quantitative, with a pre-experimental design, carried out with 16 students. The instrument
\end{abstract}


was validated by expert judgment and through statistical analysis: SPSS, version 27, giving the respective reliability. The results show that the "plucked string method" was effective for basic guitar learning in groups; there was a significant improvement; In the pre-test, $75 \%$ of the students were at a low level, $6.8 \%$ had a very low level and $18.3 \%$ were at a regular level. After applying the program, the post test indicates that $25 \%$ had a regular level, $43.8 \%$ were at a good level and $25 \%$ achieved a level of excellence. In conclusion, the referred method was effective for learning music and playing the guitar. Keywords: method, execution, guitar, group learning.

\section{Resumo}

A presente investigação tem como objetivo determinar a eficácia do método das cordas dedilhadas, para a aprendizagem básica do violão em grupo. Esta pesquisa é quantitativa, com desenho pré-experimental, realizada com 16 alunos. $\mathrm{O}$ instrumento foi validado por julgamento de especialistas e por meio de análise estatística: SPSS, versão 27, conferindo a respectiva confiabilidade. Os resultados mostram que o "método das cordas dedilhadas" foi eficaz para o aprendizado básico do violão em grupos; houve uma melhora significativa; No pré-teste, $75 \%$ dos alunos estavam em nível baixo, 6,8\% em nível muito baixo e 18,3\% em nível regular. Após a aplicação do programa, o pós-teste indica que $25 \%$ tiveram um nível regular, $43,8 \%$ um bom nível e $25 \%$ um nível de excelência. Concluindo, o referido método foi eficaz para o aprendizado de música e tocar violão.

Palavras-chave: método, execução, violão, aprendizagem em grupo.

\section{Introducción}

La presencia de la guitarra en el panorama musical actual, en diversas formaciones y estilos, tanto como solista o acompañante, ha suscitado un creciente interés. Desde el siglo XX hasta esta parte, la música se ha visto en vuelta en transformaciones (Martínez, 2001). Mediante la música, desarrollan diferentes habilidades cognoscitivas dentro de estas el lenguaje, un medio importante para desarrollar la integración; habilidades físicas y sobre todo habilidades sociales, las cuales le servirán para desenvolverse adecuadamente en su entorno, además lo ayudarán en su vida futura (Montoya, 2016). Asimismo, cabe mencionar la posibilidad para superar paulatinamente sus temores, ansiedades y dificultades que se presenten. 
El aprendizaje musical es un proceso sumamente complejo, que exige el desarrollo de habilidades específicas: auditivas, de ejecución y de creación en tiempo real o diferido. A la vez, se apoya en la asimilación de contenidos -conceptos, hechos, proposiciones, sistemas teóricos- y el fomento de actitudes, propios de cada praxis musical (Rusinek, 2004). El desarrollo de las capacidades musicales es necesario tanto para el aprendizaje "de base" de la escolarización obligatoria como para el aprendizaje vocacional, en las escuelas de música, y el profesional, en los conservatorios (Molina, 2011).

En el desarrollo de la Estimulación Musical presenta un hecho recurrente respecto a falta de material educativo en el área de música (Alave, 2016). Esta falta de textos escolares obliga a los docentes de música a preparar un programa especial acorde a las necesidades de la institución educativa y al grado para el que se necesita (Bustos-Ríos, 2014).

En el Perú, no se elaboran textos escolares para música, esta necesidad obliga a los docentes a preparar su propio material de trabajo para poder realizar una enseñanza de calidad (Deodanes Belloso \& Flores Vásquez, 2012). Esta debe ser específica al instrumento musical que se desea desarrollar (Vicente, 2009). Sabemos que en diferentes instituciones privadas se usa la flauta dulce, en otras el violín, también instrumentos de viento y percusión que conforman la banda de música (trompeta, tuba, saxofón, clarinete, flauta traversa, tambor, bombo, tarola, platillos, etc.).

En respuesta a la problemática antes planteada se desarrolló un programa, para el aprendizaje básico de la guitarra de manera grupal en los estudiantes. Asimismo, se desarrolló las habilidades y la capacidad para mejorar la concentración y disciplina, contribuyendo en gran medida para el desempeño exitoso de las personas en su vida académica y profesional. Este planteamiento tiene mucha importancia en todos los ámbitos de la vida y para enfrentar las competencias académicas en este siglo se debe promover la creatividad en expresar sus emociones. El programa se realizó a través de sesiones, exposiciones, videos y demostraciones, con el propósito de mejorar el aprendizaje guitarrístico.

\section{Técnicas de la guitarra}

La historia de la música nos demuestra su influencia magnífica, como transmisora de esencias sonoras hispánicas". Así mismo, el gran guitarrista y compositor Emilio Pujol se refiere a la gran importancia del desarrollo de una buena técnica para lograr alcanzar 
con éxito las metas trazadas de poder interpretar en el instrumento las músicas que deseemos.

Debemos entender por técnica de un instrumento musical la manera correcta y más adecuada de ejecutar estudios y obras escritas para ese instrumento (Soto, 2018), prosigue diciendo que la técnica instrumental marca la diferencia entre un buen ejecutante y otro que lo hace con deficiencia. Carlevaro (1979) presenta magistralmente las diferentes maneras de pulsar la guitarra y acompaña en sus cuatro cuadernos las escalas mayores y menores, arpegios muy variados para el desarrollo técnico de la mano derecha y ejercicios para la mano izquierda, tales como: desplazamiento, sustitución, saltos, ligados, dedos fijos para la independencia, ceja, etc. Siendo uno de los materiales obligados para todo aquel que desee aprender a ejecutar bien el instrumento en mención.

\section{Lectura y ejecución de melodías}

Podemos afirmar que todos los métodos para guitarra poseen una sección dedicada a la lectura y ejecución de melodías, esto es debido a que en el orden lógico del aprendizaje de este instrumento es uno de los primeros pasos a seguir. Este método presenta en la primera parte lectura musical de melodías, el autor quiere que los estudiantes se familiaricen con la ejecución melódica del instrumento, seguidamente aborda el trabajo didáctico por tonalidades presentando los acordes de la tonalidad, la escala y un ejercicio melódico para reforzar la lectura musical en la posición a ejecutar.

\section{Guitarra rítmica o de acompañamiento.}

La guitarra es más conocida por sus bondades armónicas para el acompañamiento, podemos observar que la gran mayoría de jóvenes y adultos que tocan guitarra conocen los acordes de la guitarra y la usan como instrumento rítmico y de acompañamiento. Es así como los métodos antes mencionados como "Voz y Guitarra" de Junek y Casas están enfocados, además de la lectura de melodías, al estudio del acompañamiento.

Se ha creado una infinidad de libros, revistas y folletos que enseñan a los interesados en la guitarra a usar toda esta gama de acordes para acompañar una canción. Podemos citar, a manera de ejemplo al método "How to play guitar" del pedagogo musical Roger Evans, donde presenta diferentes círculos armónicos para acompañar.

También se ha elaborado una versión del Himnario Adventista con acordes para guitarra, con la finalidad de que los jóvenes amantes de la guitarra puedan acompañar todos los himnos. De la misma forma existen miles de músicas con cifrado para guitarra. 


\section{Guitarra solística.}

Esta forma de ejecutar la guitarra es la más elaborada y compleja, demanda muchos años de estudio y es la que lleva a la formación de concertistas. En nuestra propuesta esta guitarra nos permite interpretar solos fáciles con una polifonía principalmente a dos voces, buscando que el estudiante ejecute himnos religiosos del Himnario Adventista y algunos solos de música tradicional, peruana y clásica.

\section{Lenguaje musical.}

El lenguaje musical es muy importante, permite la lectoescritura de la música en lo que llamamos partitura. Molina (2011) presenta los símbolos musicales como el lenguaje de cualquier idioma, con sus letras que permite escribir y leer. Él lo presenta como un todo que contiene ritmo y pulso formando una melodía. El lenguaje musical abarca: teoría, lectura rítmica, solfeo hablado, solfeo entonado y audio perceptiva.

\section{Teoría musical}

La teoría es importante, fundamenta la base de un conocimiento. De Rubertiz (1937) presenta una manera fácil de comprender los conceptos de la música a través de preguntas y respuestas, exponiendo de manera sencilla y clara todos los conocimientos que el estudiante debe saber.

Actualmente, con el internet también encontramos páginas de fácil acceso a la teoría musical, una de ellas es teoría.com, donde el estudiante puede encontrar respuestas a todas sus interrogantes. Explica todos los elementos que el estudiante debe conocer y menciona la evolución natural de la música desde la antigüedad.

\section{Lectura rítmica}

Presenta el tiempo musical desde sus comienzos y el trabajo de lectura con el empleo del metrónomo (instrumento para medir el ritmo). En este material encontramos ejercicios y teoría vinculados con el pulso, acento y ritmo. Ha proporcionado ejercicios importantes para mejorar nuestro aporte. A través del programa "cuerda pulsada" presentamos ejercicios rítmicos progresivos extraídos de las músicas que se están estudiando, así el alumno puede saber la duración de cada figura musical en el contexto de la obra. 


\section{Solfeo hablado}

El artista e interprete musical Bona nos presenta un material ideal, para la lectura hablada en clave de sol, ideal para el estudiante de guitarra. En este libro encontramos las notas musicales en el pentagrama y con diferentes ritmos, para ejercitar el reconocimiento de las notas, llevando un pulso y ritmo correcto.

\section{Solfeo entonado}

Hemos creído conveniente utilizar las mismas melodías a ejecutar en la guitarra, para realizar la parte correspondiente a la lectura entonada. Esto permite que el alumno interiorice las melodías y sean más fáciles de comprender e interpretar en la guitarra. Cada melodía aprendida es, a la vez, un ejercicio de lectura entonada (Zambrano, 2002).

\section{Audio perceptiva}

Este es un aspecto que permite al alumno reconocer si una melodía sube, baja o se mantiene en la misma altura. La guitarra es un instrumento armónico, desde el inicio del estudio, todo estudiante está empleando acordes mayores y menores; por consiguiente, aprende a reconocer la brillantez y alegría de los acordes mayores, la tristeza y melancolía de los acordes menores y los sonidos preparatorios, para el cambio armónico de los acordes de séptima dominante.

\section{Metodología}

Corresponde a un diseño pre experimental, permitió la aplicación del programa "Cuerda pulsada", para evidenciar su efecto y relación con una o más variables dependientes. Asimismo, se aplicó una pre y post prueba, aplicadas a un solo grupo (Hernández et al., 2014). La población fue de 16 estudiantes del primer año de secundaria del Colegio Unión de Ñaña. El proceso de muestreo no es probabilístico, sino intencional y por conveniencia.

El instrumento de recolección de datos fue elaborado por el investigador de esta tesis, el cual es una guía, cuyo fin es evaluar el nivel guitarrístico, con las respectivas validaciones. La matriz de evaluación está conformada con un total de 12 ítems y 4 dimensiones, distribuidas de la siguiente manera: 1. Ejecución de melodías, 4 ítems; 2. Guitarra rítmica o de acompañamiento, 4 ítems, 3. Guitarra solística, 4 ítems y, 4. Lectura musical, 4 ítems. 
Para la recolección de datos se siguió los siguientes procesos: después de contar con la autorización de la institución educativa, se procedió a tomar el examen de preprueba del programa “Cuerda pulsada”. Luego se pasó a dictar las 18 sesiones de clase, correspondientes al todo el proceso del aprendizaje básico de la guitarra de manera grupal, durante el tiempo establecido. El tratamiento estadístico de la información se procesó con el programa SPSS, versión 27 en español. Todos los datos recogidos se analizaron mediante la estadística descriptiva e inferencial; para mostrar los resultados (expresados en porcentajes, promedios y desviaciones típicas o estándar) se utilizaron tablas univariadas y bivariadas.

\section{Resultados}

\section{Nivel de conocimiento musical antes y después del programa}

Tabla 1

Nivel de conocimiento musical en evaluación antes del programa "Cuerda Pulsada"

\begin{tabular}{lll}
\hline Niveles & Frecuencia & Porcentaje \\
\hline Muy Bajo & 12 & $75 \%$ \\
Bajo & 1 & $6.3 \%$ \\
Regular & 3 & $18.8 \%$ \\
Total & 16 & $100 \%$ \\
\hline
\end{tabular}

En la tabla 1 se aprecia que la mayoría de los estudiantes presenta un nivel muy bajo (75\%) en conocimiento musical. Asimismo, existe un grupo (18.8\%) que presenta un nivel regular de conocimiento musical, siendo este el mejor nivel alcanzado.

Tabla 2

Nivel de conocimiento musical en evaluación después del programa "Cuerda Pulsada"

\begin{tabular}{lll}
\hline Niveles & Frecuencia & Porcentaje \\
\hline Bajo & 1 & $6,3 \%$ \\
Regular & 4 & $25,0 \%$ \\
Bueno & 4 & $25,0 \%$ \\
Excelente & 7 & $43,8 \%$ \\
Total & 16 & 100 \\
\hline
\end{tabular}


En la tabla 2 se aprecia que la mayoría de los estudiantes presenta un nivel excelente $43,8 \%$ y un nivel bueno el $25,0 \%$ de los estudiantes. Otro $25,0 \%$ un nivel regular y sólo el $6.3 \%$ presenta un nivel bajo en conocimiento musical. No se percibe el nivel muy bajo, los participantes han mejorado notablemente su nivel de conocimiento musical.

\section{Análisis comparativo por el género}

Tabla 3

Análisis comparativo entre el aprendizaje guitarrístico y el género

\begin{tabular}{lllllll}
\hline & \multicolumn{2}{c}{ Antes } & & & & Total \\
\cline { 2 - 7 } Género & Muy & Bajo & Regular & Bueno & Excelente & \\
& bajo & & & & & \\
Masculino & $75,0 \%$ & $6,3 \%$ & $18,8 \%$ & $0,0 \%$ & $0,0 \%$ & $100 \%$ \\
Femenino & $82,6 \%$ & $8,4 \%$ & $0,0 \%$ & $0,0 \%$ & $0,0 \%$ & $100 \%$ \\
Total & $85 \%$ & $10 \%$ & $5,0 \%$ & $0,0 \%$ & $0,0 \%$ & $100 \%$ \\
\hline
\end{tabular}

La tabla 3 muestra que el género femenino tuvo un mejor aprovechamiento del programa, respecto al masculino. En la evaluación de entrada, el 75,0\% de varones se encontraba en muy bajo y el $6.3 \%$ en bajo. Por su parte, el género femenino el $82.6 \%$ se encontraba en nivel muy bajo y el $8,4 \%$ en nivel bajo, respectivamente. No obstante, después de la aplicación del programa "Cuerda pulsada", el 90\% de varones subió en el nivel bueno y el 10\% alcanzó el nivel excelente, mientras que el 85\% de las mujeres alcanzó el nivel excelente.

Tabla 4

Análisis comparativo entre la ejecución de melodías y el género

\begin{tabular}{llllllllllllll}
\hline & Antes & \multicolumn{1}{c}{ Total } & Después & & Total \\
\cline { 2 - 11 } Género & $\begin{array}{l}\text { Muy } \\
\text { bajo }\end{array}$ & Bajo & Regular & Bueno & Excelente & & $\begin{array}{c}\text { Muy } \\
\text { bajo }\end{array}$ & Bajo & Regular & Bueno & Excelente \\
\hline Masculino & $85.4 \%$ & $0,0 \%$ & $14,6 \%$ & $0,0 \%$ & $0,0 \%$ & $100 \%$ & $0,0 \%$ & $0,0 \%$ & $0,0 \%$ & $15 \%$ & $85 \%$ & $100 \%$ \\
Femenino & $72.1 \%$ & $13,9 \%$ & $0,0 \%$ & $0,0 \%$ & $0,0 \%$ & $100 \%$ & $0,0 \%$ & $0,0 \%$ & $0,0 \%$ & $5 \%$ & $95 \%$ & $100 \%$ \\
Total & $85 \%$ & $5,0 \%$ & $10,0 \%$ & $0,0 \%$ & $0,0 \%$ & $100 \%$ & $0,0 \%$ & $0,0 \%$ & $0,0 \%$ & $15 \%$ & $85 \%$ & $100 \%$ \\
\hline
\end{tabular}

La tabla 4 muestra que el género femenino tuvo un mejor aprovechamiento del programa, respecto al masculino. En la evaluación de entrada, el 85,4\% de varones se 
encontraba en nivel muy bajo y el 14,6\% en nivel regular. Por su parte, el género femenino el $72,1 \%$ se encontraba en nivel muy bajo y el $13,9 \%$ en nivel bajo respectivamente. No obstante, después de la aplicación del programa "Cuerda pulsada", el 85\% de varones subió en el nivel excelente y el 15\% alcanzó el nivel bueno, mientras que el 95\% de las mujeres alcanzó el nivel excelente

Tabla 5

Análisis comparativo entre la guitarra rítmica o de acompañamiento y el género.

\begin{tabular}{llllllllllllll}
\hline & \multicolumn{1}{c}{ Antes } & \multicolumn{1}{c}{ Total } & Después & & Total \\
\cline { 2 - 12 } Género & $\begin{array}{l}\text { Muy } \\
\text { bajo }\end{array}$ & Bajo & Regular & Bueno & Excelente & $\begin{array}{c}\text { Muy } \\
\text { bajo }\end{array}$ & Bajo & Regular & Bueno & Excelente \\
\hline Masculino & $95 \%$ & $5,0 \%$ & $0,0 \%$ & $0,0 \%$ & $0,0 \%$ & $100 \%$ & $0,0 \%$ & $0,0 \%$ & $0,0 \%$ & $8 \%$ & $92 \%$ & $100 \%$ \\
Femenino & $100 \%$ & $0,0 \%$ & $0,0 \%$ & $0,0 \%$ & $0,0 \%$ & $100 \%$ & $0,0 \%$ & $0,0 \%$ & $0,0 \%$ & $0,0 \%$ & $100 \%$ & $100 \%$ \\
Total & $95 \%$ & $5,0 \%$ & $0,0 \%$ & $0,0 \%$ & $0,0 \%$ & $100 \%$ & $0,0 \%$ & $0,0 \%$ & $0,0 \%$ & $4 \%$ & $96 \%$ & $100 \%$ \\
\hline
\end{tabular}

La tabla 5 muestra que el género femenino tuvo un mejor aprovechamiento del programa, respecto al masculino. En la evaluación de entrada, el 95\% de varones se encontraba en nivel muy bajo y el 5,0\% en nivel bajo. Por su parte, el género femenino el $100 \%$ se encontraba en nivel muy bajo. No obstante, después de la aplicación del programa "Cuerda pulsada", el 92\% de varones subió en el nivel excelente y el 8\% alcanzó el nivel bueno, mientras que el 96\% de las mujeres alcanzó el nivel excelente.

Tabla 6

Análisis comparativo entre la guitarra solística y el género.

\begin{tabular}{llllllllllllll}
\hline & Antes & \multicolumn{1}{c}{ Total } & Después & & Total \\
\cline { 2 - 12 } Género & $\begin{array}{l}\text { Muy } \\
\text { bajo }\end{array}$ & Bajo & Regular & Bueno & Excelente & & $\begin{array}{c}\text { Muy } \\
\text { bajo }\end{array}$ & Bajo & Regular & Bueno & Excelente \\
\hline Masculino & $96.4 \%$ & $4,6 \%$ & $0,0 \%$ & $0,0 \%$ & $0,0 \%$ & $100 \%$ & $0,0 \%$ & $0,0 \%$ & $0,0 \%$ & $7.6 \%$ & $92.4 \%$ & $100 \%$ \\
Femenino & $100 \%$ & $0,0 \%$ & $0,0 \%$ & $0,0 \%$ & $0,0 \%$ & $100 \%$ & $0,0 \%$ & $0,0 \%$ & $0,0 \%$ & $11.8 \%$ & $88.2 \%$ & $100 \%$ \\
Total & $97 \%$ & $3,0 \%$ & $0,0 \%$ & $0,0 \%$ & $0,0 \%$ & $100 \%$ & $0,0 \%$ & $0,0 \%$ & $0,0 \%$ & $14 \%$ & $86 \%$ & $100 \%$ \\
\hline
\end{tabular}

La tabla 6 muestra que el género femenino tuvo un mejor aprovechamiento del programa, respecto al masculino. En la evaluación de entrada, el 96,4\% de varones se encontraba en nivel muy bajo y el 4,6\% en nivel bajo. Por su parte, el género femenino el $100 \%$ se encontraba en nivel muy bajo. No obstante, después de la aplicación del 
programa "Cuerda pulsada", el 92.4\% de varones subió en el nivel excelente y el 7,6\% alcanzó el nivel bueno, mientras que el 86\% de las mujeres alcanzó el nivel excelente.

Tabla 7

Análisis comparativo entre la lectura musical y el género.

\begin{tabular}{|c|c|c|c|c|c|c|c|c|c|c|c|c|}
\hline \multirow[b]{2}{*}{ Género } & \multicolumn{5}{|c|}{ Antes } & \multirow[t]{2}{*}{ Total } & \multicolumn{5}{|c|}{ Después } & \multirow[t]{2}{*}{ Total } \\
\hline & $\begin{array}{l}\text { Muy } \\
\text { bajo }\end{array}$ & Bajo & Regular & Bueno & Excelente & & $\begin{array}{l}\text { Muy } \\
\text { bajo }\end{array}$ & Bajo & Regular & Bueno & Excelente & \\
\hline Masculino & $97 \%$ & $3,0 \%$ & $0,0 \%$ & $0,0 \%$ & $0,0 \%$ & $100 \%$ & $0,0 \%$ & $0,0 \%$ & $0,0 \%$ & $17 \%$ & $83 \%$ & $100 \%$ \\
\hline Femenino & $94 \%$ & $6,0 \%$ & $0,0 \%$ & $0,0 \%$ & $0,0 \%$ & $100 \%$ & $0,0 \%$ & $0,0 \%$ & $0,0 \%$ & $8.7 \%$ & $91.3 \%$ & $100 \%$ \\
\hline Total & $93 \%$ & $7,0 \%$ & $0,0 \%$ & $0,0 \%$ & $0,0 \%$ & $100 \%$ & $0,0 \%$ & $0,0 \%$ & $0,0 \%$ & $14 \%$ & $86 \%$ & $100 \%$ \\
\hline
\end{tabular}

La tabla 7 muestra que el género femenino tuvo un mejor aprovechamiento del programa, respecto al masculino. En la evaluación de entrada, el 97\% de varones se encontraba en nivel muy bajo y el 3,0\% en nivel bajo. Por su parte, el género femenino el $94 \%$ se encontraba en nivel muy bajo y el $6,0 \%$ en nivel bajo respectivamente. No obstante, después de la aplicación del programa "Cuerda pulsada", el 83\% de varones subió en el nivel excelente y el 17\% alcanzó el nivel bueno, mientras que el $86 \%$ de las mujeres alcanzó el nivel excelente.

\section{Prueba de normalidad}

Tabla 8

Prueba de normalidad - Ajuste de bondad

\begin{tabular}{llll}
\hline & \multicolumn{2}{l}{ Shapiro - Will } \\
\cline { 2 - 4 } & Estadístico & Gl & Sig. \\
Preprueba &, 940 & 16 &, 552 \\
Postprueba &, 9299 & 16 &, 375 \\
\hline
\end{tabular}

La tabla 8 muestra la prueba de Bondad de Ajuste del pre y post prueba del aprendizaje básico de la guitarra de manera grupal. Los resultados obtenidos evidencian que el Sig. 0,552 de la preprueba y el Sig. 0,375 de la posprueba, son mayores que 0,05; por lo tanto, se establece que los datos provienen de una distribución normal; por lo cual, para el análisis de los datos, se utilizó estadígrafos paramétricos 


\section{Prueba de Wilcoxon de la variable ejecución de melodías}

Tabla 9

Prueba de Wilcoxon de la variable ejecución de melodías.

\begin{tabular}{ll}
\hline & Preprueba - Posprueba \\
\hline Z & $-3,537$ \\
Sig.asisntót. (bilateral) &, 000 \\
\hline
\end{tabular}

a. Prueba de rangos con signo de Wilcoxon

b. Se basa en rangos negativos.

En la tabla 9 se observa que el valor de (Z) es de $\mathbf{- 3 , 5 3 7}$ y el valor de significancia es.0,000 $(\boldsymbol{p}<\mathbf{0 . 0 0})$; por lo tanto, se rechaza la hipótesis nula y se acepta la hipótesis alterna. En efecto, queda demostrado que el método "Cuerda pulsada" mejoró significativamente la dimensión ejecución de melodías en los estudiantes.

\section{Prueba de Wilcoxon de la variable guitarra rítmica o de acompañamiento.}

Tabla 10

Prueba de Wilcoxon de la variable guitarra rítmica o de acompañamiento.

\begin{tabular}{ll}
\hline & Preprueba - Posprueba \\
\hline$Z$ & $-3,542$ \\
Sig.asisntót. (bilateral) &, 000 \\
\hline
\end{tabular}

a. Prueba de rangos con signo de Wilcoxon

b. Se basa en rangos negativos.

En la tabla 10 se observa que el valor de (Z) es de $\mathbf{- 3 , 5 4 2}$ y el valor de significancia es.0,000 $(\boldsymbol{p}<\mathbf{0 . 0 0})$; por lo tanto, se rechaza la hipótesis nula y se acepta la hipótesis alterna. En efecto, queda demostrado que el método "Cuerda pulsada" mejoró significativamente la dimensión guitarra rítmica o de acompañamiento en los estudiantes. 


\section{Prueba de Wilcoxon de la variable guitarra solística}

Tabla 11

Prueba de Wilcoxon de la variable guitarra solística.

\begin{tabular}{ll}
\hline & Preprueba - Posprueba \\
\hline Z & $-3,538$ \\
Sig.asisntót. (bilateral) &, 000 \\
\hline
\end{tabular}

a. Prueba de rangos con signo de Wilcoxon

b. Se basa en rangos negativos.

En la tabla 11 se observa que el valor de $(Z)$ es de $\mathbf{- 3 , 5 3 8}$ y el valor de significancia es.0,000 $(\boldsymbol{p}<\mathbf{0 . 0 0})$; por lo tanto, se rechaza la hipótesis nula y se acepta la hipótesis alterna. En efecto, queda demostrado que el método "Cuerda pulsada" mejoró significativamente la dimensión guitarra solística en los estudiantes.

\section{Prueba de Wilcoxon de la variable lectura musical}

Tabla 12

Prueba de Wilcoxon de la variable lectura musical.

\begin{tabular}{ll}
\hline & Preprueba - Posprueba \\
\hline Z & $-3,532$ \\
Sig.asisntót. (bilateral) &, 000 \\
\hline
\end{tabular}

a. Prueba de rangos con signo de Wilcoxon

b. Se basa en rangos negativos.

En la tabla 12 se observa que el valor de $(Z)$ es de $\mathbf{- 3 , 5 3 2}$ y el valor de significancia es.0,000 $(\boldsymbol{p}<\mathbf{0 . 0 0})$; por lo tanto, se rechaza la hipótesis nula y se acepta la hipótesis alterna. En efecto, queda demostrado que el método "Cuerda pulsada" mejoró significativamente la dimensión lectura musical en los estudiantes.

\section{Conclusiones}

El programa "Cuerda Pulsada" es eficaz, para el aprendizaje básico de la guitarra de manera grupal, en los estudiantes del primer año de secundaria del Colegio Unión de 
Ñaña, 2019, según los resultados obtenidos, cuyo promedio de la prueba de entrada es 20.00, el de la prueba de salida es 83.00

En cuanto a la dimensión de la ejecución de melodía, según los resultados obtenidos, cuyo promedio de la prueba de entrada es 5.7, el de la prueba de salida es 17.82. Asimismo, en la dimensión guitarra rítmica, según los resultados obtenidos, cuyo promedio de la prueba de entrada es 5.73, el de la prueba de salida es 16.27. Del mismo modo, en la dimensión solística, según los resultados obtenidos, cuyo promedio de la prueba de entrada es 1.55, el de la prueba de salida es 18.98. Finalmente, en la dimensión lectura musical, según los resultados obtenidos, cuyo promedio de la prueba de entrada es 1.65 , el de la prueba de salida es 17.68 .

\section{Referencias}

Alave, C. G. (2016). Efectividad del Programa "Yo amo mi música” en el desarrollo de la Estimulación Musical de los estudiantes del Primer Grado de Educación Primaria de la Institución Educativa Particular Peruano Americano UGEL 06, 2014 [[Tesis para la obtención del título profesional, Universidad Peruana Unión]]. https://repositorio.upeu.edu.pe/handle/20.500.12840/370

Bustos-Ríos, P. R. (2014). Situación de la Enseñanza Musical impartida por docentes de Educación Inicial de la ciudad de Cuenca, año lectivo 2012 - 2013 [Tesis de magister, Universidad de Cuenca]. http://dspace.ucuenca.edu.ec/bitstream/123456789/21093/1/tesis.pdf.pdf

Carlevaro, A. (1979). Escuela de Guitarra Exposición de la teoría instrumental. Barry Etlitorial.

https://www.academia.edu/4821184/Abel_Carlevaro_Escuela_de_la_guitarra

De Rubertiz. (1937). Teoría completa de la música. RICORDI. http://www.imae.edu.uy/images/emuse/Teoría de la Música Parte 1.pdf

Deodanes Belloso, M. E., \& Flores Vásquez, S. I. (2012). Las Técnicas de Oralidad en el Proceso Penal Salvadoreño y la importancia del Contrainterrogatorio en el desarrollo de la Vista Pública [Tesis, Universidad de El Salvador]. http://repositorioslatinoamericanos.uchile.cl/handle/2250/159333

Hernández, R., Fernández, C., \& Baptista, P. (2014). Metodología de la investigación 
(Sexta aedi). McGraw Hill Interamericana Editores, S.A. de C.V. https://doi.org/10.1017/CBO9781107415324.004

Martínez, M. C. (Ed.). (2001). Aprendizaje de la argumentación razonada. Desarrollo temático de los textos expositivos y argumentativos (Segunda ed). Unidad de Artes Gráficas de la Facultad de Humanidades de la Universidad del Valle. https://www.academia.edu/29470390/Aprendizaje_de_la_argumentación_razonad $\underline{\mathrm{a}}$

Molina, E. (2011). Hacer música... para aprender a componer: composición en grupo. Eufonía: Didáctica de La Música, 10(51), 53-64. https://dialnet.unirioja.es/servlet/articulo?codigo $=3583523$

Montoya, I. N. (2016). La educación musical en la integración de niños con necesidades educativas especiales [Tesis de licenciatura, Pontificia Universidad Católica del Perú]. https://tesis.pucp.edu.pe/repositorio/handle/20.500.12404/7920

Rusinek, G. (2004). Aprendizaje musical significativo. Revista Electrónica Complutense de Investigación en Educación Musical, 1(5), 1-17. https://d1wqtxts1xzle7.cloudfront.net/66934079/Aprendizaje_musical_significativ o20210504-23600-shsd72-with-cover-page-

v2.pdf?Expires $=1640102681 \&$ Signature $=$ SIUj3RThMvuP54022RxPmJxXCreKX xUJmT-LcTyeg8P54WhOJVrjHxgx0H86VGz-

\section{j14ud6XAgFckyXnfZJ08syQqg3hBqrEzI4p5U}

Soto, P. S. (2018). La técnica de la guitarra clásica en el chile del siglo xx. Aportes para una historia crítica [Teis doctoral, Pontíifica Universidad Católica de Chile]. https://repositorio.uc.cl/handle/11534/22090

Vicente, G. (2009). Movimiento y danza en Educación Musical: un análisis de los libros de texto de Educación Primaria [Tesis doctoral, Universidad de Murcia]. https://digitum.um.es/digitum/handle/10201/10046

Zambrano, D. J. (2002). Estrategias didácticas para promover la sensibilidad por la música en el primer grado de la escuela primaria. [Tesis de maestría, Universidad Autónoma de Nuevo León]. http://eprints.uanl.mx/5143/1/1020147492.pdf 